\title{
Study of photon dominated regions in Cepheus B
}

\author{
B. Mookerjea ${ }^{\star}$, C. Kramer, M. Röllig, and M. Masur
}

KOSMA, I. Physikalisches Institut, Universität zu Köln, Zülpicher Strasse 77, 50937 Köln, Germany

Received 9 February 2006 / Accepted 9 June 2006

\begin{abstract}
Aims. The aim of the paper is to understand the emission from the photon dominated regions in Cepheus B, estimate the column densities of neutral carbon in bulk of the gas in Cepheus B and to derive constraints on the factors which determine the abundance of neutral carbon relative to $\mathrm{CO}$.

Methods. This paper presents $15^{\prime} \times 15^{\prime}$ fully sampled maps of [C I $]$ at $492 \mathrm{GHz}$ and ${ }^{12} \mathrm{CO} 4-3$ observed with KOSMA at $1^{\prime}$ resolution. The new observations have been combined with the FCRAO ${ }^{12} \mathrm{CO} 1-0$, IRAM-30 ${ }^{13} \mathrm{CO} 2-1$ and $\mathrm{C}^{18} \mathrm{O} 1-0$ data, and far-infrared continuum data from HIRES/IRAS. The KOSMA- $\tau$ spherical PDR model has been used to understand the [CI] and CO emission from the PDRs in Cepheus B and to explain the observed variation of the relative abundances of both $\mathrm{C}^{0}$ and $\mathrm{CO}$.

Results. The emission from the PDR associated with Cepheus B is primarily at $V_{\mathrm{LSR}}$ between -14 and $-11 \mathrm{~km} \mathrm{~s}^{-1}$. We estimate about $23 \%$ of the observed [C II] emission from the molecular hotspot is due to the ionized gas in the HII region. Over bulk of the material the $\mathrm{C}^{0}$ column density does not change significantly, $(2.0 \pm 1.4) \times 10^{17} \mathrm{~cm}^{-2}$, although the CO column density changes by an order of magnitude. The observed $\mathrm{C} / \mathrm{CO}$ abundance ratio varies between 0.06 and 4 in Cepheus $\mathrm{B}$. We find an anti-correlation of the observed $\mathrm{C} / \mathrm{CO}$ abundance ratio with the observed hydrogen column density, which holds even when all previous observations providing $\mathrm{C} / \mathrm{CO}$ ratios are included. Here we show that this observed variation of $\mathrm{C} / \mathrm{CO}$ abundance with total column density can be explained only by clumpy PDRs consisting of an ensemble of clumps. At high $\mathrm{H}_{2}$ column densities high mass clumps, which exhibit low $\mathrm{C} / \mathrm{CO}$ abundance, dominate, while at low column densities, low mass clumps with high $\mathrm{C} / \mathrm{CO}$ abundance dominate.
\end{abstract}

Key words. ISM: clouds - ISM: dust, extinction - ISM: H II regions - ISM: structure

\section{Introduction}

Photon Dominated Regions (PDRs) are predominantly neutral, atomic and molecular regions where the physical and chemical processes are dominated by Far Ultraviolet (FUV) radiation (cf. Sternberg \& Dalgarno 1995; Hollenbach \& Tielens 1997) and the major carbon-bearing species changes from $\mathrm{C}^{+}$through $\mathrm{C}^{0}$ to $\mathrm{CO}$. While FUV photons $(6 \mathrm{eV}<h v<13.6 \mathrm{eV})$ are primarily responsible for the heating of the cloud surfaces via photoelectric effect on dust grains, at larger depths of about 10 mag into the clouds cosmic ray induced heating dominates. Owing to the clumpy nature of the molecular clouds, contrary to the initial ideas, PDRs are not strictly confined to the surface of the molecular clouds. Rather the extended [C II] and [C I] emission observed from allover the molecular clouds suggest formation of PDRs deep inside the molecular clouds, at the surfaces of clumps inundated with FUV photons escaping into the cloud. Further, the chemical abundances of several key species like e.g., $\mathrm{CN}$ and $\mathrm{HCN}$ depend to a large extent on the abundances of $\mathrm{C}^{0}$ and $\mathrm{C}^{+}$in the gas phase (Boger \& Sternberg 2005). PDRs may therefore be seen as the most ubiquitous phase of the ISM, observations of which together with proper modeling provide insight into the physical conditions within the molecular clouds. On a more global scale, PDRs provide direct insight into the chemical evolution of the ISM deluged with FUV photons and PDR models suggest radiation-induced feedback mechanisms that may regulate star formation rates and the column density of gas through giant molecular clouds.

* Present address: Department of Astronomy, University of Maryland, College Park, MD 20742, USA.
To the south of the Cepheus OB3 association of early-type stars lies the giant Cepheus molecular cloud, at a distance of about 730 pc (Blaauw 1964). Cepheus B, with a size of 4 pc, is the hottest ${ }^{12} \mathrm{CO}$ component (Sargent 1977, 1979) and is located near the northwestern edge of the cloud, adjacent to the H II region S155 (Sharpless 1959). The interface between the molecular cloud and the OB stars, is delineated by the optically visible $\mathrm{H}$ II region $\mathrm{S} 155$, whose very sharp edges clearly indicate the presence of ionization fronts bounding the dust/molecular cloud. The OB association itself consists of two subgroups of different ages, with the youngest lying closer to the molecular cloud (Sargent 1979). Felli et al. (1978) presented radio continuum observations which confirmed that the molecular cloud Cepheus B is surrounded by an ionization front driven by the radiation field of the bright stars that are part of the youngest generation of the Cepheus B OB3 association. The structure of the diffuse ionized gas and its energy balance can be accounted for by the UV radiation coming from the brightest members of the OB3 association (viz., HD 217086 and 217061). The physical association between the Cepheus B molecular cloud and the S155 H II region was also confirmed by the $\mathrm{H}_{2} \mathrm{CO}$ and recombination line observations of Panagia \& Thum (1981). Minchin et al. (1992) used a combination of low- $J$ CO and far-infrared (FIR) continuum observations to show that the Cepheus B molecular cloud terminates at its north-west edge with a hotspot. The hotspot lies $1^{\prime}$ to the east of the peak in $\mathrm{CO}$ column density. The high temperature of the hotspot and the associated FIR emission cannot be explained by only considering the stars in the OB3 association. It was further proposed by Minchin et al. (1992) and later confirmed using radio and near-infrared (NIR) continuum 
Table 1. Parameters of the observed transitions used to study the PDRs in Cepheus B.

\begin{tabular}{llll}
\hline \hline Species & Transition & $\begin{array}{l}E_{\mathrm{u}} / \mathrm{k} \\
(\mathrm{K})\end{array}$ & $\begin{array}{l}n_{\mathrm{cr}} \\
\left(\mathrm{cm}^{-3}\right)\end{array}$ \\
\hline$[\mathrm{C} \mathrm{I}]$ & ${ }^{3} \mathrm{P}_{1} \rightarrow{ }^{3} \mathrm{P}_{0}$ & 23.6 & $1 \times 10^{3}$ \\
${ }^{12} \mathrm{CO}$ & $1-0$ & 5.6 & $4.8 \times 10^{3}$ \\
${ }^{13} \mathrm{CO}$ & $2-1$ & 16.8 & $3.2 \times 10^{4}$ \\
${ }^{12} \mathrm{CO}$ & $4-3$ & 56 & $2.6 \times 10^{5}$ \\
\hline
\end{tabular}

observations by Testi et al. (1995) that the hotspot is indeed energized by a highly reddened main sequence star (NIR-A) of spectral type B1-B0.5, together with a small cluster of young stars.

In a first study with the KOSMA $3 \mathrm{~m}$ telescope, Beuther et al. (2000) mapped the Cepheus B cloud in 2-1 and 3-2 transitions of ${ }^{12} \mathrm{CO},{ }^{13} \mathrm{CO}$, and $\mathrm{C}^{18} \mathrm{O}$ at $2^{\prime}$ resolution and used PDR models of Störzer et al. (2000) to interpret the emission at four selected positions. The hotspot emission indicated the presence of shocks. Based on the derived local volume densities of $\sim 2 \times 10^{4} \mathrm{~cm}^{-3}$, and average volume density less than $10^{3} \mathrm{~cm}^{-3}$, Beuther et al. (2000) concluded that Cepheus B is highly clumped with clumps filling only $2 \%$ to $4 \%$ by volume of the cloud. In this work [C I] at $492 \mathrm{GHz}$ was detected from two selected positions and detection of [C I emission from deep inside the cloud was also put forward as an additional evidence for the clumpiness of Cepheus B.

In clumpy or homogeneous PDR scenario both [C I] and ${ }^{12} \mathrm{CO}$ 4-3 emission arise from regions closer to the surfaces of the FUV irradiated clumps or molecular cloud surfaces respectively. Thus the [C I] and ${ }^{12} \mathrm{CO} 4-3$ emission trace similar regions. While $[\mathrm{CI}]$ emission necessarily implies photodissociation of molecular $\mathrm{CO}$, the higher kinetic temperature (the upper energy level being $56 \mathrm{~K}$ ) required to excite the ${ }^{12} \mathrm{CO} 4-3$ line suggest origin of this emission from the outer surfaces of dense ( since the critical density of the ${ }^{12} \mathrm{CO} 4-3$ transition for collisions with $\mathrm{H}_{2}$ is $\sim 10^{5} \mathrm{~cm}^{-3}$ ) clumps embedded in a UV radiation field. Thus in order to trace the PDR we have observed largescale $\left(15^{\prime} \times 15^{\prime}\right)$ fully-sampled maps of the [C I] and ${ }^{12} \mathrm{CO} 4-3$ emission from the Cepheus B molecular cloud. At a common resolution of $1^{\prime}$, this dataset has been combined with FCRAO ${ }^{12} \mathrm{CO}$ $1-0$, IRAM-30 $\mathrm{m}^{13} \mathrm{CO} 2-1$ and $\mathrm{C}^{18} \mathrm{O} 1-0$ data, and FIR continuum data from HIRES/IRAS. This allows for a detailed analysis of the PDRs in Cepheus B, since observed transitions span a substantial range of critical densities and temperatures (Table 1).

\section{Datasets}

\subsection{KOSMA observations of PDR tracers: $\mathrm{C}$ I and ${ }^{12} \mathrm{Co} 4-3$}

We have used the KOSMA $3 \mathrm{~m}$ submillimeter telescope on Gornergrat, Switzerland (Winnewisser et al. 1986; Kramer et al. 1998) to observe the emission of fine-structure line of neutral carbon at $492 \mathrm{GHz}\left(609 \mu \mathrm{m},{ }^{3} \mathrm{P}_{1}-{ }^{3} \mathrm{P}_{0}\right.$; hereafter [C I] $)$ and $J=4-$ 3 transition of ${ }^{12} \mathrm{CO}$ emission at $461 \mathrm{GHz}$ from the PDRs associated with Cepheus B. We used the Submillimeter Array Receiver for Two frequencies (SMART) (Graf et al. 2002) on KOSMA for these observations. SMART is a dual-frequency eight-pixel SIS-heterodyne receiver capable of observing simultaneously in the two atmospheric windows centered around $650 \mu \mathrm{m}$ and $350 \mu \mathrm{m}$. The IF signals were analyzed with array-acoustooptical spectrometers with a spectral resolution of $1.5 \mathrm{MHz}$ (Horn et al. 1999).
The observations were performed in the position-switched On-The-Fly mode between November 2004 and March 2005. The HPBW of the [CI] and ${ }^{12} \mathrm{CO} 4-3$ observations are $60^{\prime \prime}$ with a beam efficiency of $\eta_{\mathrm{mb}}=50 \%$. Atmospheric calibration was done by measuring the atmospheric emission at the OFF-position to derive the opacity (Hiyama 1998). Sideband imbalances were corrected using standard atmospheric models (Cernicharo 1985). All data presented in this paper are in units of main beam temperature $\left(T_{\mathrm{mb}}\right)$, calculated from the observed calibrated antenna temperature $\left(T_{\mathrm{A}}{ }^{*}\right)$ using the derived beam efficiencies $\left(\eta_{\mathrm{mb}}\right)$ for the relevant telescope, $T_{\mathrm{mb}}=T_{\mathrm{A}}^{*} / \eta_{\mathrm{mb}}$. From observations of reference sources we estimate the relative calibration accuracy to be $\sim 15 \%$. Data was reduced using the GILDAS $^{1}$ astronomical data reduction package.

\subsection{Further CO data}

We have complemented the [CI] and ${ }^{12} \mathrm{CO} 4-3$ datasets for Cepheus B with low- $J$ CO observations available in literature. We have obtained the ${ }^{12} \mathrm{CO} 1-0$ dataset from the FCRAO outer Galaxy survey (Heyer et al. 1998) and ${ }^{13} \mathrm{CO} 2-1$ and $\mathrm{C}^{18} \mathrm{O} 1-0$ datasets observed with the IRAM $30 \mathrm{~m}$ by Ungerechts et al. (2000) in 1995 . The beam efficiency, $\eta_{\mathrm{mb}}$ (defined as $B_{\text {eff }} / F_{\text {eff }}$ ) used to derive the $T_{\mathrm{mb}}$ scale are $0.45,0.48$ and 0.75 for the ${ }^{12} \mathrm{CO} 1-0,{ }^{13} \mathrm{CO} 2-1$ and $\mathrm{C}^{18} \mathrm{O} 1-0$ lines respectively (Heyer et al. 1998; Kramer \& Wild 1994). All datasets have been smoothed to a resolution of $1^{\prime}$ for comparison with our observations. These $\mathrm{CO}$ datasets provide deeper insight into the physical conditions of the regions emitting $[\mathrm{C} \mathrm{I}]$ and ${ }^{12} \mathrm{CO} 4-3$ emission. ${ }^{12} \mathrm{CO} 1-0$ being optically thick, is most likely to be arising from closer to the PDR surface. The ${ }^{13} \mathrm{CO} 2-1$ and $\mathrm{C}^{18} \mathrm{O} 1-0$ on the other hand are most likely to be optically thin and hence arise from deeper inside the clumps, and hence are crucial for ascertaining the physical parameters (like the optical extinction) characterizing the emitting regions.

\subsection{HIRES processed IRAS maps}

Dust continuum emission accounts for about $98 \%$ of the cooling of the molecular clouds. Here we have used FIR continuum maps to estimate the intensity of the FUV radiation field crucial for the chemical and physical structure of the PDRs. We have obtained HIRES-processed (Aumann et al. 1990) IRAS maps of Cepheus B at 60 and $100 \mu \mathrm{m}$ from the Infrared Processing and Analysis Center, Caltech. These dust continuum maps have angular resolutions (1'.5) comparable to our submillimeter datasets.

\section{Observational results}

\subsection{Integrated intensity maps}

Figure 1a shows the integrated intensity maps of [CI] and ${ }^{12} \mathrm{CO}$ 4-3 emission from Cepheus B. The maps are centered at $\alpha=22^{\mathrm{h}} 57^{\mathrm{m}} 25^{\mathrm{s}} .7 \delta=62^{\circ} 34^{\prime} 40^{\prime} \cdot 7$ (J2000). The ${ }^{12} \mathrm{CO} 4-3$ emission peaks at the position of the hotspot, and drops off significantly towards the south-east, and does not show much substructures. The $[\mathrm{CI}]$ emission in contrast though rather weak at the position of the hotspot, has almost uniform intensity over the ridge extended along the east-west direction with an isolated peak to the south-west (below the hotspot) and an extended high intensity peak deep into the molecular cloud to the east of the

\footnotetext{
${ }^{1}$ http://www.iram.fr/IRAMFR/GILDAS
} 



Fig. 1. Velocity-integrated intensity maps of $[\mathrm{CI}]{ }^{3} \mathrm{P}_{1}-{ }^{3} \mathrm{P}_{0}$ emission (color) overlayed with contours of a) ${ }^{12} \mathrm{CO} 4-3$, b) ${ }^{13} \mathrm{CO} 2-1$, c) ${ }^{12} \mathrm{CO} 1-0$ and d) $\mathrm{C}^{18} \mathrm{O} 1-0$ intensities. All maps are integrated over the range $V_{\mathrm{LSR}}=-20$ to $-5 \mathrm{~km} \mathrm{~s}^{-1}$. Colorscale of the [C I] map and contour levels of all other line intensities are indicated at the top left corner of each panel. The red star denotes the position of the hot spot. All data are shown at a common resolution of $1^{\prime}$.

map. The somewhat different distribution of ${ }^{12} \mathrm{CO} 4-3$ and [C I] emission suggest that while the ${ }^{12} \mathrm{CO} 4-3$ emission traces higher temperature and densities as in the hotspot, the $[\mathrm{CI}]$ emission being optically thin traces the embedded PDR surfaces of the molecular material with higher column density better. The features seen in the ${ }^{12} \mathrm{CO} 4-3$ map agree reasonably well with the features seen in the ${ }^{12} \mathrm{CO} 3-2$ map by Beuther et al. (2000).

Figures $1 \mathrm{~b}, \mathrm{c}$ and $\mathrm{d}$ show overlays of the observed [C I] emission with the ${ }^{13} \mathrm{CO} 2-1,{ }^{12} \mathrm{CO} 1-0$ and $\mathrm{C}^{18} \mathrm{O} 1-0$ intensities respectively. The $[\mathrm{CI}]$ and ${ }^{13} \mathrm{CO} 2-1$ appear to be almost concomitant tracing similar features in the Cepheus B cloud. To the west of the map the ${ }^{12} \mathrm{CO} 1-0$ emission is similar in parts with the $[\mathrm{CI}]$ emission. However the ${ }^{12} \mathrm{CO} 1-0$ intensities drop off more drastically towards the east, which is similar to the features seen in the ${ }^{12} \mathrm{CO} 4-3$ map. This is probably due to the higher column densities to the east, as manifested by the peaks in the ${ }^{13} \mathrm{CO}$ and $\mathrm{C}^{18} \mathrm{O}$ maps. The lower ${ }^{12} \mathrm{CO} 4-3$ emission to the east, stem from a combination of lower densities and kinetic temperatures. The $\mathrm{C}^{18} \mathrm{O} 1-0$ emission, tracing higher column densities, looks markedly different from the ${ }^{12} \mathrm{CO} 4-3$ emission and almost similar to the $[\mathrm{C} \mathrm{I}]$ emission except for the region close to the hotspot where, the $\mathrm{C}^{18} \mathrm{O}$ emission is almost absent. The faint ${ }^{13} \mathrm{CO} 2-1$ emission and almost no $\mathrm{C}^{18} \mathrm{O} 1-0$ emission from the hotspot is consistent with the results of Minchin et al. (1992), who found a distinct lack of ${ }^{13} \mathrm{CO}$ emission at the position of the hotspot.

\subsection{Velocity structure}

Previous low- $J$ CO observations showed that the Cepheus B emission consists of two major velocity components: one, the north-western part of the cloud closer to the hot spot emits at $\sim-15 \mathrm{~km} \mathrm{~s}^{-1}$, confirming the association of the hot spot with S155 having the same system velocity and second, the southeastern part emitting at $\sim-12 \mathrm{~km} \mathrm{~s}^{-1}$ (Beuther et al. 2000; Minchin et al. 1992).

An overlay of the [CI] and ${ }^{12} \mathrm{CO} 4-3$ channel maps (Fig. 2) shows that bulk of the cloud emits between -14 and $-11 \mathrm{~km} \mathrm{~s}^{-1}$, although substructures contributing to the ${ }^{12} \mathrm{CO} 4-3$ emission change from the hotspot to the clump to the south-west of the 


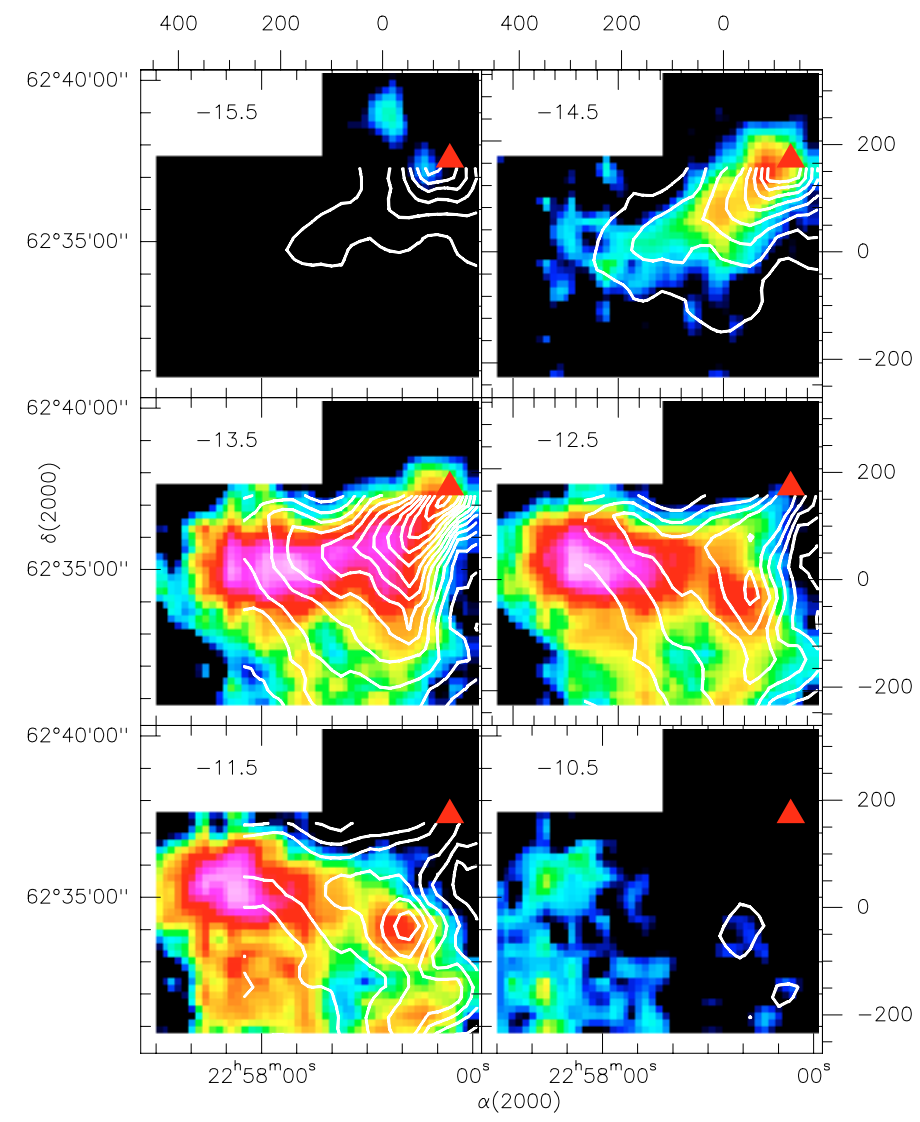

Fig. 2. Overlay of $[\mathrm{CI}]$ (color) and ${ }^{12} \mathrm{CO} \quad 4-3$ (contours) velocity channel maps of Cepheus B. The colorscale ranges between 1 and $11.6 \mathrm{~K} \mathrm{~km} \mathrm{~s}^{-1}$ and the ${ }^{12} \mathrm{CO} \mathrm{4-3}$ contours are between 5 and $120 \mathrm{~K} \mathrm{~km} \mathrm{~s}^{-1}$ in steps of $15 \mathrm{~K} \mathrm{~km} \mathrm{~s}^{-1}$.

Table 2. Velocity components derived from Gaussian fitting of spectra at the position of the hotspot in Cepheus B.

\begin{tabular}{lll}
\hline \hline Line & $\begin{array}{l}V_{\mathrm{LSR}} \\
\left(\mathrm{km} \mathrm{s}^{-1}\right)\end{array}$ & $\begin{array}{l}F W H M \\
\left(\mathrm{~km} \mathrm{~s}^{-1}\right)\end{array}$ \\
\hline$[\mathrm{C} \mathrm{I}]$ & -14.1 & 1.9 \\
${ }^{12} \mathrm{CO} \mathrm{4-3}$ & -13.9 & 2.8 \\
${ }^{12} \mathrm{CO} \mathrm{1-0}$ & -13.6 & 2.2 \\
${ }^{13} \mathrm{CO} 2-1$ & -13.4 & 2.1 \\
{$[\mathrm{C} \mathrm{II}$ Comp 1 } & -13.6 & 3.8 \\
{$[\mathrm{C}$ II $]$ Comp 2 } & -16.5 & 6.0 \\
\hline
\end{tabular}

map. The emission from the hotspot is slightly blue-shifted, between -16 and $-14 \mathrm{~km} \mathrm{~s}^{-1}$. At $-15.5 \mathrm{~km} \mathrm{~s}^{-1}$, we detect in [C I] a clump to the extreme north of the map. At $-10.5 \mathrm{~km} \mathrm{~s}^{-1}$ the ${ }^{12} \mathrm{CO} 4-3$ emission from the cloud is too low and the [C I] emission is restricted mainly to the east of the ridge. It is interesting to note that at $-11.5 \mathrm{~km} \mathrm{~s}^{-1}$ the $[\mathrm{CI}]$ and ${ }^{12} \mathrm{CO} 4-3$ emission from the south-western clump are exactly concomitant.

Figure 3 shows the [CI], CO and [C II] $158 \mu \mathrm{m}$ spectra observed at the position of the hotspot. The [C II] spectrum was observed by Boreiko et al. (1990) using the Kuiper Airborne Observatory with a resolution of 43", similar to the resolution of the other spectra $\left(60^{\prime \prime}\right)$. The velocity resolution is $0.8 \mathrm{~km} \mathrm{~s}^{-1}$. It is one of the very few heterodyne spectra of the [C II] line taken so far. These spectra show that at the position of the hotspot the PDR emission is dominated by the velocity component at $V_{\mathrm{LSR}}$ of $-13.8 \mathrm{~km} \mathrm{~s}^{-1}$ and the lineshapes with the exception of [C II] are all primarily Gaussian. The [C II] line at the hotspot

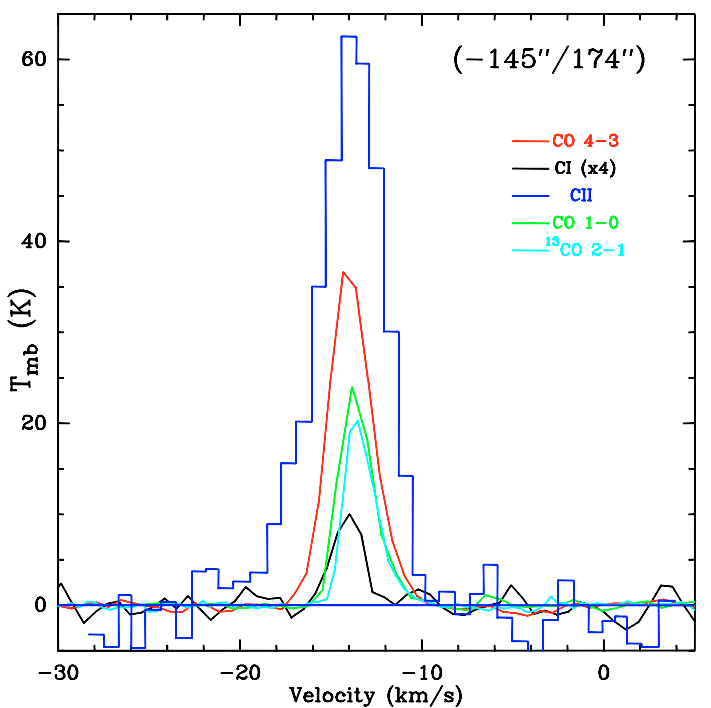

Fig. 3. Observed $\mathrm{CI},{ }^{12} \mathrm{CO} 4-3,{ }^{12} \mathrm{CO} 1-0,{ }^{13} \mathrm{CO} 2-1$ and [C II] spectra at the position of the molecular hotspot in Cepheus B. The [C II] spectrum is adopted from Boreiko et al. (1990). The ratio of integrated intensities ${ }^{12} \mathrm{CO} 4-3 /{ }^{12} \mathrm{CO} 1-0$ at this position is 1.9 . All data are shown at a common resolution of $1^{\prime}$, except for the [C II] data which has a resolution of $43^{\prime \prime}$.

position appears to be the broadest, with traces of an extended blue wing. Boreiko et al. (1990) have explained the [C II] emission from the hotspot position to be from two velocity components, the main narrow component is centered at $-13.6 \mathrm{~km} \mathrm{~s}^{-1}$ with a width of $3.8 \mathrm{~km} \mathrm{~s}^{-1}$, while the blue component is centered at $-16.8 \mathrm{~km} \mathrm{~s}^{-1}$ and has a width of $6.0 \mathrm{~km} \mathrm{~s}^{-1}$. Table 2 presents results of fitting Gaussian profiles to the different spectral lines observed at the hotspot. Broadly we find that both [C I] and $\mathrm{CO}$ emission arise around $V_{\mathrm{LSR}}$ of $-13.8 \mathrm{~km} \mathrm{~s}^{-1}$ and have linewidths $\sim 2.2 \mathrm{~km} \mathrm{~s}^{-1}$. We have reproduced the Gaussian fits to the [C II] spectra obtained by Boreiko et al. (1990) using the same parameters. The broader blue component at $-16.5 \mathrm{~km} \mathrm{~s}^{-1}$ does not appear to be associated with the emission from the PDR/molecular cloud at all, rather the velocity matches the $V_{\mathrm{LSR}}$ shown by $\mathrm{H} \alpha$ emission from the H II region S155 (Boreiko et al. 1990). Based on the Gaussian fit to the [C II] spectrum, we estimate $\sim 77 \%$ of the measured [C II] emission arises from the PDR and the remaining from the ionized gas. The estimated relative contribution of PDRs to [C II] emission from nearby galaxies using the [N II] $122 \mu \mathrm{m}$ is $\sim 70-85 \%$ (Malhotra et al. 2001; Kramer et al. 2005), roughly consistent with the above result for Cepheus B.

\section{LTE analysis}

\subsection{The $C O(4-3) / C O(1-0)$ distribution}

Figure $4 \mathrm{a}$ shows a map of the ratio of the ${ }^{12} \mathrm{CO} 4-3 /{ }^{12} \mathrm{CO} 1-0$ intensities integrated over the velocity range between -20 and $-5 \mathrm{~km} \mathrm{~s}^{-1}$ for the region in Cepheus B. It shows a smooth gradient between ratios of $0.3-0.6$ in the south-east and ratios of $1.5-2.2$ to the north-west of the mapped region.

Ratios of $0.9 \pm 0.2$, as seen towards the bulk of the molecular ridge, suggest that both transitions are thermalized and can be explained in terms of LTE and optically thick emission. Neglecting the Rayleigh-Jeans correction, the ratio is expected to be 1.0 in this case. The calibration uncertainty of line ratios is $\sim 20 \%$. The $4-3 / 1-0$ ratio is sensitive to excitation temperatures 

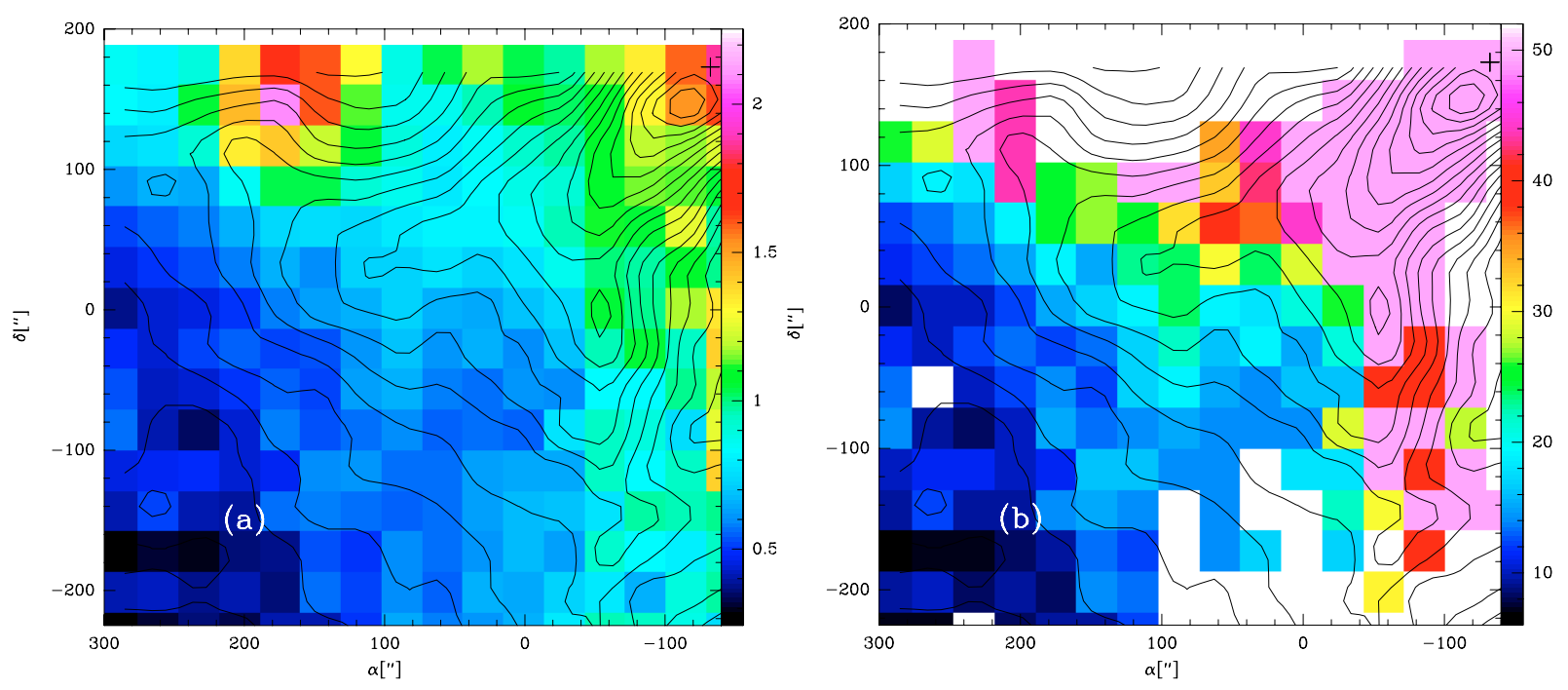

Fig. 4. a) Distribution of observed ${ }^{12} \mathrm{CO} 4-3 /{ }^{12} \mathrm{CO} 1-0$ ratio in Cepheus B. b) Distribution of excitation temperatures $T_{\text {ex }}$ derived from the ${ }^{12} \mathrm{CO}$ 4-3/1-0 line ratio of integrated intensities, assuming LTE. Contours in both panels correspond to integrated intensities of ${ }^{12} \mathrm{CO} 4-3$.

only upto $50 \mathrm{~K}$, beyond that the ratio remains almost constant with increasing $T_{\mathrm{ex}}$.

While ratios less than 0.8 suggest sub-thermal excitation of ${ }^{12} \mathrm{CO} 4-3$, ratios above 1.2 are inconsistent with above assumptions. At the position of the hotspot for which the spectra are shown in Fig. 3 the integrated intensity ratio $4-3 / 1-0$ has a value of 1.9. Similar to this position, at all other positions with high 4-3/1-0 ratios, we do not see any evidence of self absorption of either line by cold foreground gas. Within the framework of single $T_{\text {ex }}$ and LTE, differences in optical thickness and volume filling factors of the emitting regions of the two lines could still partially explain the observed high $4-3 / 1-0$ ratios. However we consider strong outwardly increasing temperature gradients to be the more plausible explanation. Such a gradient results in an inconsistency in deriving a single $T_{\mathrm{ex}}$ from this ratio, since the 4-3 transition will predominantly trace the warm, still dense, outer regions, while the $1-0$ traces the somewhat inner, colder regions. Such high ${ }^{12} \mathrm{CO}$ ratios are typically found in the surface regions of molecular clouds subject to strong UV fields (Castets et al. 1990; Kramer et al. 1996; Beuther et al. 2000; Kramer et al. 2004).

Excitation temperatures derived from the 4-3/1-0 line ratios vary smoothly between $\sim 8 \mathrm{~K}$ in the south-east of the mapped region and more than $50 \mathrm{~K}$ in the north-west near the position of the hot core and at the western edge of the molecular cloud (Fig. 4b). In the following discussion, we have assumed a constant excitation temperature of $50 \mathrm{~K}$ for $4-3 / 1-0>0.9$.

\section{2. $C^{0} / C O$ abundances in Cepheus $B$}

We have estimated the $\mathrm{C}^{0}$ and $\mathrm{CO}$ column densities based on LTE approximation. For these calculations we have used the observed [C I] and $\mathrm{C}^{18} \mathrm{O} 1-0$ line intensities, the $T_{\text {ex }}$ estimated from the ${ }^{12} \mathrm{CO} 4-3 /{ }^{12} \mathrm{CO} 1-0$ ratios and a relative abundance ratio $[\mathrm{CO}] /\left[\mathrm{C}^{18} \mathrm{O}\right]$ of 500 (Wilson \& Rood 1994). Further we have assumed both $[\mathrm{CI}]$ and $\mathrm{C}^{18} \mathrm{O} 1-0$ to be optically thin. The more detailed PDR analysis presented in Sect. 6, strongly supports this latter assumption.

Assuming LTE $N(\mathrm{CO})$ depends linearly on $T_{\text {ex }}$ above $20 \mathrm{~K}$, while in contrast, $N(\mathrm{C})$ is rather independent of the assumed $T_{\mathrm{ex}}$ to within $20 \%$ between 20 and $150 \mathrm{~K}$. However, in between 10 and $20 \mathrm{~K}, N(\mathrm{C})$ drops by more than a factor of 2 , while $N(\mathrm{CO})$ remains constant within $30 \%$.

Figure 5a shows a scatterplot of the the $\mathrm{C}^{0}$ column densities, $N(\mathrm{C})$ versus the $\mathrm{CO}$ column densities, $N(\mathrm{CO})$. CO column densities vary by more than one order of magnitude between $10^{17}$ and $2 \times 10^{18} \mathrm{~cm}^{-2}$, corresponding to optical extinctions between $\sim 1 \mathrm{mag}$ and $30 \mathrm{mag}$. Although the range of values of $N(\mathrm{C})$ at different positions appear to be similar to the range shown by $N(\mathrm{CO})$, a histogram of $N(\mathrm{C})$ shows that for most positions $N(\mathrm{C})$ does not show much variation, staying almost constant at a mean value of $2.0 \times 10^{17} \mathrm{~cm}^{-2}$ with an rms of $1.4 \times 10^{17} \mathrm{~cm}^{-2}$.

Figure $5 \mathrm{~b}$ shows a map of the $N(\mathrm{C}) / N(\mathrm{CO})(\mathrm{C} / \mathrm{CO}$ hereafter $)$ overlayed with contours of integrated intensities of $\mathrm{C}^{18} \mathrm{O}$. This plot shows that regions of higher $\mathrm{C}^{18} \mathrm{O} 1-0$ intensities, presumably tracing high $\mathrm{H}_{2}$ column densities, show rather low values of $\mathrm{C} / \mathrm{CO}$, in contrast to the outskirts. The ridge and the southwestern core show low values of $\mathrm{C} / \mathrm{CO}(\sim 0.1)$, while the ratio rises gradually to values up to 4 outside these regions where the $\mathrm{CO}$ emission is more diffuse.

The constancy of $\mathrm{C}^{0}$ column densities is also reflected in Fig. 6 showing a scatterplot of the $\mathrm{C} / \mathrm{CO}$ abundance ratio versus $\mathrm{H}_{2}$ column densities or optical extinctions derived from $N(\mathrm{CO})$ assuming a $\mathrm{CO} / \mathrm{H}_{2}$ abundance of $8 \times 10^{-5}$ (Blake et al. 1987). The $\mathrm{C} / \mathrm{CO}$ ratio is $\sim 1$ for $2 \mathrm{mag}$ and drops to 0.1 for $20 \mathrm{mag}$. A linear fit to the data results in

$\log \left[N\left(\mathrm{C}^{0}\right) / N(\mathrm{CO})\right]=(-1.01 \pm 0.03) N\left(\mathrm{H}_{2}\right)+(21.5 \pm 0.72)$

with a correlation coefficient of -0.82 . The slope is almost -1 as would be expected for nearly constant $N(\mathrm{C})$.

Figure 6 also shows the $\mathrm{C} / \mathrm{CO}$ abundances derived in other Galactic star forming regions and diffuse clouds. These studies cover a range of optical extinctions between $0.3 \mathrm{mag}$ and $\sim 100$ mag with $\mathrm{C} / \mathrm{CO}$ abundances varying by more than two orders of magnitude between $\sim 4$ and $\sim 10^{-2}$, approximately following the slope we find in Cepheus B.

Clearly, the [C I] 1-0 line is not a straightforward tracer of optical extinctions or total $\mathrm{H}_{2}$ column densities and total masses.

The scatter of $\mathrm{C} / \mathrm{CO}$ abundance varies with $A_{\mathrm{V}}$. For $A_{\mathrm{V}}$ 's upto 20 mag the scatter decreases from being almost comparable to the mean $\mathrm{C} / \mathrm{CO}$ itself to about $50 \%$ of the mean. At $\sim 20 \mathrm{mag}$ the scatter is the smallest, with the $\mathrm{C} / \mathrm{CO}$ abundance from five different data sets agreeing to a value of 0.1 with a scatter of 

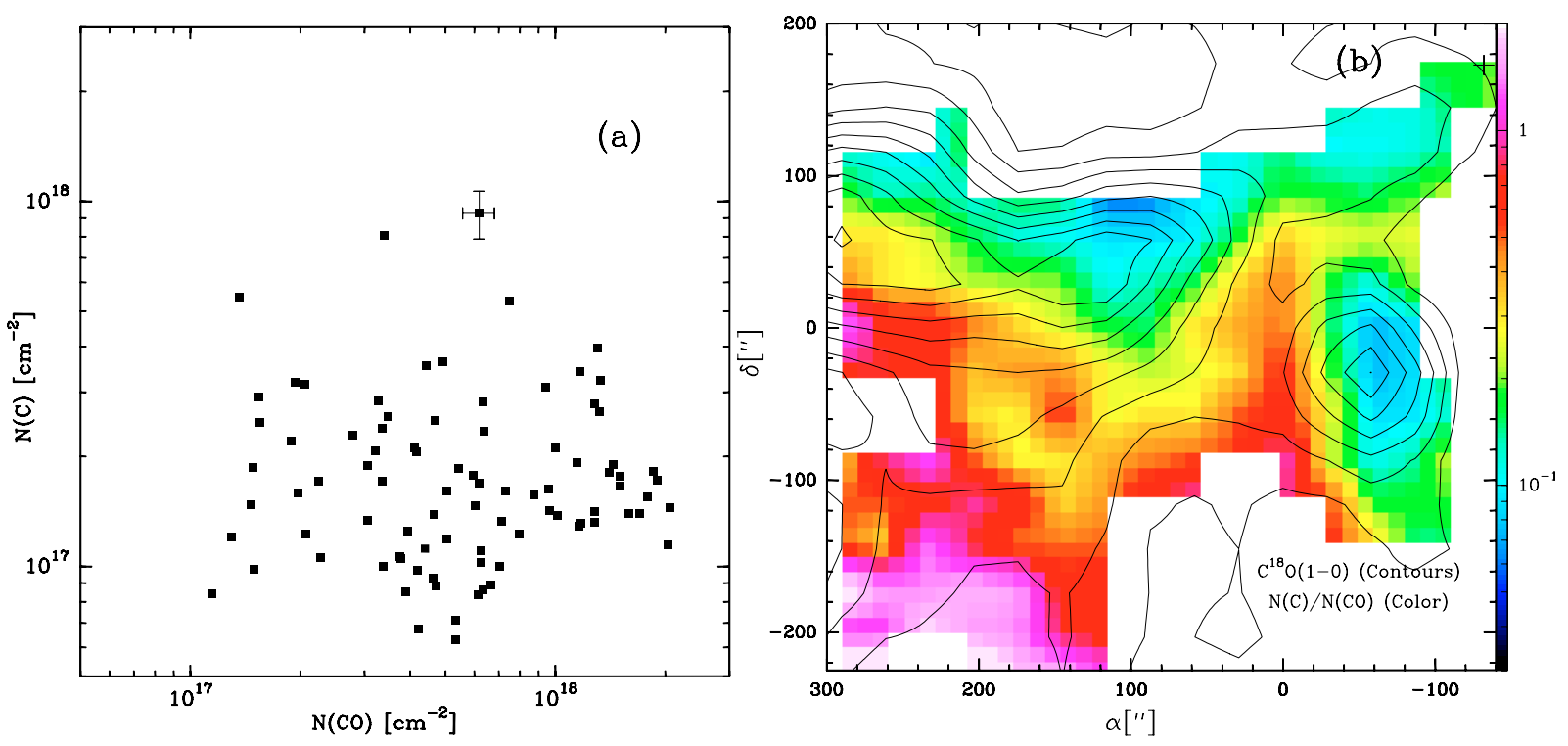

Fig. 5. a) $N(\mathrm{C})$ vs. $N(\mathrm{CO})$ for independent positions (positions separated by $1^{\prime}$, i.e., beamwidth) in the map. Typical errors of $15 \%$ for $N(\mathrm{C})$ and $10 \%$ for $N(\mathrm{CO})$ are shown for one position. b) The $\mathrm{C}^{0} / \mathrm{CO}$ abundance ratio in Cepheus B overlayed with the contours of integrated intensity of $\mathrm{C}^{18} \mathrm{O} 1-0$. We have considered here only those positions for which we have ${ }^{12} \mathrm{CO} 4-3$ data and for which the $[\mathrm{C} \mathrm{I}]$ and $\mathrm{C}^{18} \mathrm{O}$ intensities lie above 3 times their respective rms noise levels.

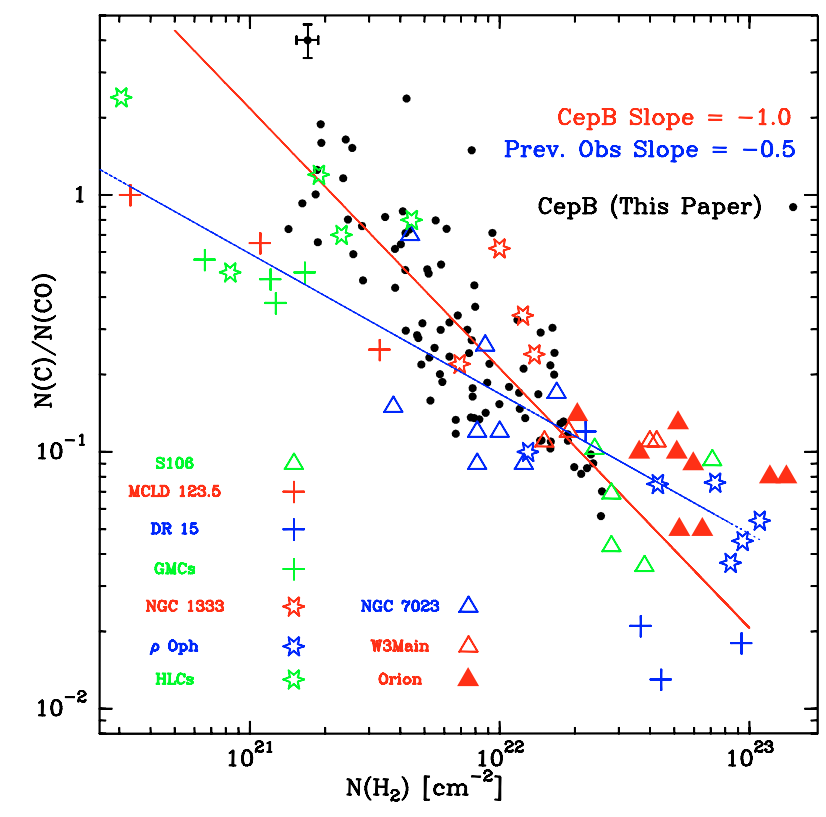

Fig. 6. Column density ratio of $\mathrm{C}$ to $\mathrm{CO}$ in Cepheus B. Plotted as in Fig. 5 are independent positions, which are separated by $1^{\prime}$. Also shown are observed values in other Galactic molecular clouds like NGC 7023 (Gerin et al. 1998), S106 (Schneider et al. 2003), DR 15 (Oka et al. 2001), MCLD 123.5+24.9 (Bensch et al. 2003), High Latitude Clouds (Ingalls et al. 1997), NGC 1333 (Oka et al. 2004), $\rho$ Oph (Kamegai et al. 2003), W3Main (Kramer et al. 2004) and Orion (Ikeda et al. 2002). The points corresponding to GMCs are taken from Plume et al. (1999) in which [CI] data of Cep A, S 140, NGC 2024 and W3 were presented. The red drawn line corresponds to the parameter derived by fitting only the Cepheus B data. Typical errors of $15 \%$ for $N(\mathrm{C}) / N(\mathrm{CO})$ and $10 \%$ for $N\left(\mathrm{H}_{2}\right)$ are shown for one position. The blue line is the result of fitting data points for all previous observations.

0.05 . Note that several studies which cover only a small range of extinctions find rather constant C/CO abundances.

In Sect. 6 we show that clumpy PDR models naturally explain the observed variation of the $\mathrm{C} / \mathrm{CO}$ abundance.

\section{FUV intensity from FIR continuum}

The spectral lines observed in Cepheus B which are used here are not sensitive to the FUV radiation field, and there are no largescale [C II] observations available in the literature for this region. We thus estimate the FUV radiation field from the observed FIR continuum. Because dust grains absorb most of the FUV photons incident on the cloud and most of that energy is reemitted in the form of FIR continuum, and other heating mechanisms like cosmic ray heating can be neglected, the intensity of the FUV radiation can be inferred from the FIR continuum. This estimate is valid even if a fraction of FUV photons escape without impinging the cloud.

We have estimated the FIR intensity of the mapped region using the HIRES processed 60 and $100 \mu \mathrm{m}$ IRAS fluxes following the relationship (Nakagawa et al. 1998):

$I_{\mathrm{FIR}}=3.25 \times 10^{-11}\left[\frac{f_{v}(60 \mu \mathrm{m})}{\mathrm{Jy} \mathrm{sr}^{-1}}\right]+1.26 \times 10^{-11}\left[\frac{f_{v}(100 \mu \mathrm{m})}{\mathrm{Jy} \mathrm{sr}^{-1}}\right]$.

The FUV intensity expressed in units of the Draine field (Draine 1978; Draine \& Bertoldi 1996) is given by

$\chi=\frac{1}{1.71} \frac{4 \pi}{\left(2 \times 1.610^{-3}\right)} I_{\mathrm{FIR}}$.

Following Kaufman et al. (1999), this formula takes into account that there is about equal heating of the grains by photons outside the FUV band (leading to the factor 2) and that the bolometric dust continuum emission is a factor of $\sim 2$ larger than the FIR intensity derived above from the IRAS fluxes. We also assume that the emission stems from slabs which are illuminated from all sides and the FIR emission stems from the back and front sides. The latter two corrections approximately cancel out. Finally, in order to convert the FUV field expressed in units of Habing field $\left(1.6 \times 10^{-3} \mathrm{erg} \mathrm{s}^{-1} \mathrm{~cm}^{-2}\right.$; Habing 1968) to Draine field it is required to divide by 1.71 .

Figure 7 shows the distribution of FUV intensity calculated as above, overlayed with the observed [C I] emission. Within the region mapped, the FUV field varies smoothly between $\sim 25$ in 


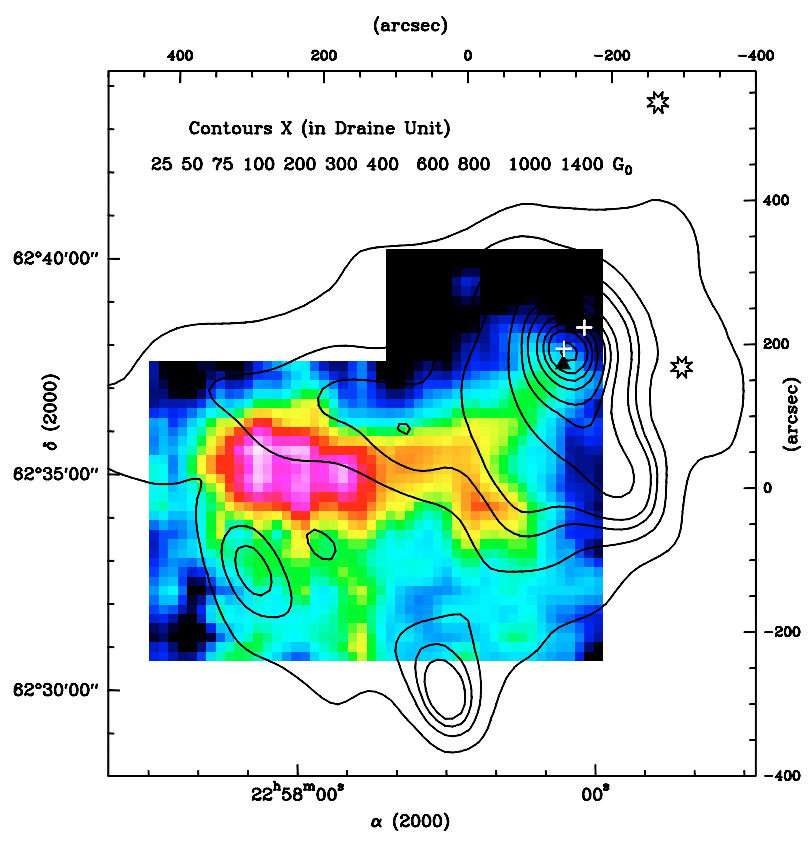

Fig. 7. FUV intensity distribution (red contours) in Cepheus B estimated from the FIR fluxes measured by IRAS, overlayed with [C I] integrated intensities (in color). Contour levels of the FUV intensity are indicated at the top left corner. The intensity scale for the [C I] map are the same as in Fig. 1. White stars denote HD 217086 (north) and HD 217061, Crosses denote the NIR sources A-NIR (south) and B-NIR and the filled triangle denotes the location of the hot spot.

the south-east and 1500 times Draine field, in the north-west near the hot spot. This variation of $\chi$ correlates well with the corresponding variation of excitation temperatures derived from the ${ }^{12} \mathrm{CO}$ line ratios (Fig. 5b).

In Fig. 7 we have marked the positions of the nearest $\mathrm{O}$ and B stars, HD 217086 (north-west), HD 217061 (west) and the embedded NIR sources A and B (cf. Testi et al. 1995). NIR-A, which does not have any infrared excess and which is deemed to be a highly reddened main sequence star of spectral type B1-B0.5, is responsible for the excitation of the associated blister $\mathrm{H}$ II region (the radio continuum source A). The FUV intensity distribution calculated as above is consistent with the results of Testi et al. (1995) and suggest that the star A-NIR is mostly responsible for the FIR emission from the region. The total estimated FIR luminosity from the IRAS fluxes and for the assumed distance of $730 \mathrm{pc}$ for Cepheus B is $\sim 10^{4} L_{\odot}$ and this is consistent with the luminosity due to a B1 type star as well.

\section{PDR model interpretation of $\mathrm{C}^{0} / \mathrm{CO}$ vs. $\mathrm{N}\left(\mathrm{H}_{2}\right)$ relation}

We interpret here the observed anti-correlation of the $\mathrm{C} / \mathrm{CO}$ abundance ratio with the hydrogen column density $\left(N\left(\mathrm{H}_{2}\right)\right)$ in terms of PDRs created due to the FUV irradiation of molecular material. For this we have used the KOSMA- $\tau$ PDR model (Störzer et al. 1996; Röllig et al. 2006). This model considers spherical clouds illuminated by an isotropic FUV radiation field and cosmic rays, calculates the resulting chemical and temperature structure of the cloud. This is followed by radiation transfer calculations to estimate the emission of different species. The KOSMA- $\tau$ model has ${ }^{13} \mathrm{CO}$ chemistry included in its network. The emission from the models are calculated as a function of the hydrogen volume density, FUV radiation field and mass of the spherical clump. The KOSMA- $\tau$ model assumes that a clump



Fig. 8. Results from the PDR models for external FUV fields of $\chi=10^{3}$ a) and $\chi=10^{2}$, expressed in Draine units b). Contours show integrated intensity line ratios of $\mathrm{CI} /{ }^{12} \mathrm{CO} 4-3$ and $\mathrm{CI} /{ }^{13} \mathrm{CO} 2-1$. The range of observed values is shown: $0.3-0.7$ for $\mathrm{CI} /{ }^{13} \mathrm{CO} 2-1,0.2-0.6$ for $\mathrm{CI} /{ }^{12} \mathrm{CO} 4-3$. We consider only positions for which the $\mathrm{C} / \mathrm{CO}$ abundance ratio has been estimated. Both ratios suggest that the volume densities on an average lie between $10^{4}$ and $10^{5} \mathrm{~cm}^{-3}$.

has a power-law density profile of $n(r) \sim r^{-1.5}$ for $0.2 \leq r / r_{\mathrm{cl}} \leq 1$ and $n(r)=$ const. for $r / r_{\mathrm{cl}} \leq 0.2$. The surface density $n_{\mathrm{cl}}$ is about half the average clump density.

\subsection{Estimates of volume density}

First, we have used PDR models to derive the range of allowed hydrogen volume densities based on the observed $\mathrm{CI} /{ }^{13} \mathrm{CO} 2-1$ and $\mathrm{CI} /{ }^{12} \mathrm{CO} 4-3$ intensity ratios. At all the positions for which we have calculated $\mathrm{C} / \mathrm{CO}$ abundance ratio the $\mathrm{CI} /{ }^{13} \mathrm{CO} 2-1$ ratio varies between 0.3 and 0.7 , while the $\mathrm{CI} /{ }^{12} \mathrm{CO} 4-3$ ratio varies between 0.2 and 0.6. Based on FIR maps the FUV field in the mapped region varies between 50 and $10^{3}$ Draine units. Figure 8 shows the contours bracketing the observed two line ratios for constant FUV field and different PDR clump densities and masses. These two line ratios do not allow to discriminate between different clump masses. The best fitting clump densities lie between $2 \times 10^{4}$ and $8 \times 10^{5} \mathrm{~cm}^{-3}$, for clump masses between $10^{-2}$ and $10 M_{\odot}$. This range of densities is rather independent of the FUV field. We note that the clump masses are not very well constrained, however the intersection of the two ratio contours in Fig. 8 provide physically reasonable limits on clump masses, which have been loosely used in our subsequent analysis, but do not have any significant impact on our final results. The observed ${ }^{12} \mathrm{CO} 4-3 / 1-0$ line ratios are consistent with this interpretation.

\subsection{Discussion}

\subsubsection{Single spherical PDR model}

We first explore the single spherical PDR models described above, in order to derive the observed $\mathrm{C} / \mathrm{CO}$ vs. $N\left(\mathrm{H}_{2}\right)$ anticorrelation. As shown above, clumps with masses between $10^{-2}$ and $10 M_{\odot}$ with densities $\sim 10^{5} \mathrm{~cm}^{-3}$ reproduce the range of $[\mathrm{CI}] /{ }^{13} \mathrm{CO} 2-1$ and $[\mathrm{CI}] /{ }^{12} \mathrm{CO} 4-3$ intensity ratios observed in Cepheus B. In Fig. 9, we present the calculated radial variation of the $\mathrm{C}$ and $\mathrm{CO}$ abundances for two PDR clumps with masses of $10^{-3}$ and $1 M_{\odot}$. The surface density is fixed at $10^{5} \mathrm{~cm}^{-3}$ and the FUV field is fixed at $10^{2}$ (in Draine units), the average value derived from the HIRES data in Sect. 5.

At the surface of the PDRs carbon is fully ionized by the incident FUV radiation. Increasing depth leads to increased absorption of the FUV radiation by both gas and dust. This allows $\mathrm{C}^{+}$to recombine with electrons to form $\mathrm{C}^{0}$. At even larger depths $\mathrm{CO}$ 




Fig. 9. Abundance profiles of $\mathrm{C}^{0}$ and $\mathrm{CO}$ calculated by the KOSMA- $\tau$ PDR model for two clumps with masses $1 M_{\odot}$ and $0.001 M_{\odot}$. The $x$-axis is given in units of the relative depth $d / R_{\mathrm{tot}}$. A value of 1 denotes the center of the cloud. The FUV field strength is $10^{2}$ in Draine units.

Table 3. KOSMA- $\tau$ calculations of $\mathrm{H}_{2}, \mathrm{C}$ and $\mathrm{CO}$ column densities of spherical clumps with $n=10^{5} \mathrm{~cm}^{-3}$ and $\chi=10^{2}$ (Draine units). The column densities are for individual clumps, and not the column densities observed per beam.

\begin{tabular}{|c|c|c|c|c|c|}
\hline$M_{\mathrm{cl}}$ & $\begin{array}{l}N\left(\mathrm{H}_{2}\right)_{\mathrm{cl}} \\
10^{21} \\
\mathrm{~cm}^{-2}\end{array}$ & $\begin{array}{l}N(\mathrm{C})_{\mathrm{cl}} \\
10^{16} \\
\mathrm{~cm}^{-2} \\
\end{array}$ & $\begin{array}{l}N(\mathrm{CO})_{\mathrm{cl}} \\
10^{17} \\
\mathrm{~cm}^{-2}\end{array}$ & $\frac{N\left(\mathrm{C}^{0}\right)}{N(\mathrm{CO})} \mathrm{cl}$ & $R_{\mathrm{cl}}$ \\
\hline 10 & 31 & 23 & 79 & 0.03 & $\frac{1}{0.077}$ \\
\hline 1 & 14 & 20 & 32 & 0.06 & 0.036 \\
\hline 0.1 & 6.6 & 14 & 13 & 0.11 & 0.017 \\
\hline 0.01 & 3.3 & 8.7 & 4.0 & 0.22 & 0.008 \\
\hline 0.001 & 1.3 & 4.2 & 1.0 & 0.43 & 0.004 \\
\hline
\end{tabular}

starts to form because the photodissociating FUV photons are attenuated sufficiently leading to a chemical stratification typical of PDR models, $\mathrm{C}^{+} / \mathrm{C}^{0} / \mathrm{CO}$. The absolute depth where this transition occurs depends on the gas density $n$ and the FUV intensity $\chi$. In spherical models it also depends on the size $R_{\text {tot }}$ of the cloud since the local mean intensity $J_{v}(r)=(4 \pi)^{-1} \int I_{v}(r, \Omega) \mathrm{d} \Omega$ at a certain depth is larger for a small clump compared to a bigger one. Accordingly a clump with $M_{\mathrm{cl}}=1 M_{\odot}$ consists of a much larger CO core than a clump with $M_{\mathrm{cl}}=0.001 M_{\odot}$. The average $\mathrm{CO}$ column density increases with mass. The dependence of $N(\mathrm{C})$ on the mass is weaker. Once the clump becomes massive enough to significantly shield the photodissociating FUV photons a further increase in mass does not lead to larger $\mathrm{C}^{0}$ columns but larger CO column densities. This behavior is illustrated in Fig. 9 with the abundance of $\mathrm{CO}$ at the inner core (with relative depth $=1$ ) of the clumps being identical in both cases. Thus the $1 M_{\odot}$ clump has a much higher total fraction of $\mathrm{CO}$ and lower $\mathrm{C} / \mathrm{CO}$ than the lower mass clump. This indicates that the lines of sight having high $\mathrm{C} / \mathrm{CO}$ abundance ratios are dominated by clumps of smaller sizes.

Table 3 lists the clump-averaged $\mathrm{C}, \mathrm{CO}$ and $\mathrm{H}_{2}$ column densities of the KOSMA- $\tau$ PDR model, considering a single spherical clump. We note here that the column densities in Table 3 correspond to those calculated by the PDR model for a single clump and do not corroborate with the observed column densities per beam, as plotted in Fig. 6. The $\mathrm{C} / \mathrm{CO}$ ratios calculated

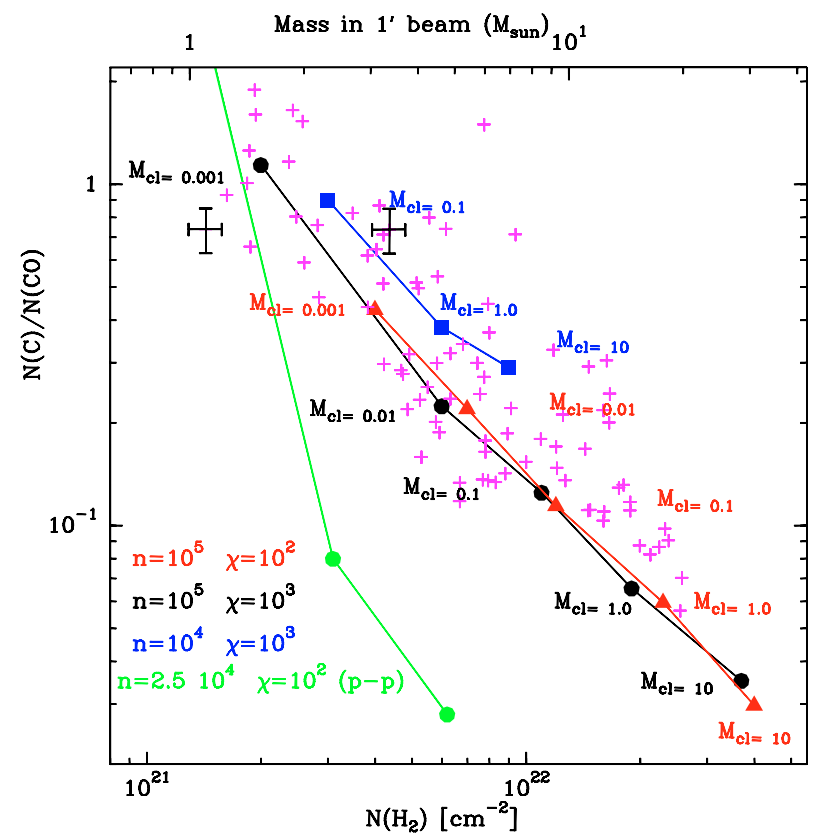

Fig. 10. KOSMA- $\tau$ PDR model interpretation of the observed $\mathrm{C} / \mathrm{CO}$ vs. $N\left(\mathrm{H}_{2}\right)$ anti-correlation. For comparison results of face-on homogeneous plane-parallel models are also shown. Drawn lines correspond to models the input parameters for which are marked at the bottom left corner. $M_{\mathrm{cl}}$ corresponds to mass of an individual clump. $M_{\text {beam }}$ shown along the upper $x$-axis is the mass of gas in the $60^{\prime \prime}$ beam. The number of clumps in the beam is given by $M_{\text {beam }} / M_{\mathrm{cl}}$.

by the PDR model is correlated with the mass of the clump. We have checked the peak optical depths of the [CI] line emission in a pencil beam centered on the clump and for the line center position. For all clumps studied here, these peak opacities are less than 0.5. The clump and line averaged opacities of the [C I] line are much less. This justifies our previous assumption, used in the LTE analysis, that the $[\mathrm{CI}]$ line is optically thin. Similarly, the modelled ${ }^{13} \mathrm{CO}$ peak opacities indicate that the $\mathrm{C}^{18} \mathrm{O}$ line is optically thin.

PDR models with $n=10^{5} \mathrm{~cm}^{-3}$ have clump sizes ranging between $0.004 \mathrm{pc}$ (for $m_{\text {clump }}=10^{-3} M_{\odot}$ ) and $0.08 \mathrm{pc}$ (for $m_{\text {clump }}=10 M_{\odot}$ ). In comparison, a beam of $60^{\prime \prime}$ corresponds to a diameter of $0.21 \mathrm{pc}$ at the distance of Cepheus B. It is thus not possible to compare the observed C/CO vs. $N\left(\mathrm{H}_{2}\right)$ per $60^{\prime \prime}$ beam correlation directly with the $N\left(\mathrm{H}_{2}\right)$ per clump $\left(N\left(\mathrm{H}_{2}\right)_{\mathrm{cl}}\right.$ in Table 3 ) obtained from the single spherical PDR clumps.

\subsubsection{The clumpy PDR scenario}

The observed uniformity of the $[\mathrm{CI}]$ intensity distribution throughout the PDR (Fig. 1) and the failure to interpret the $\mathrm{C} / \mathrm{CO}$ vs. $N\left(\mathrm{H}_{2}\right)$ using single spherical PDR model leaves open two possible physical scenarios: (i) clumpiness of the medium, i.e., either there are multiple plane-parallel slabs stacked or there is an ensemble of clumps along the line of sight, and (ii) a single, homogeneous plane-parallel PDR slab inclined relative to the major sources of UV radiation HD 217086 and HD 217061.

We first consider the simplified clumpy PDR interpretation using ensemble of clumps having the same mass and density at each position. Figure 10 shows that the observed $\mathrm{C} / \mathrm{CO}$ abundances and total $\mathrm{H}_{2}$ column densities of Cepheus B can be reproduced with clumpy PDR models. It shows results based on the ensemble interpretation using the spherical PDR models with 
Table 4. Results of PDR model calculations for ensembles of clumps having same mass and density. $\phi_{\mathrm{A}}$ is the area filling factor of the clumps.

\begin{tabular}{lllllll}
\hline \hline $\begin{array}{l}\left(n_{\mathrm{cl}}, \chi\right) \\
\left(\mathrm{cm}^{-3}, *\right)\end{array}$ & $\begin{array}{l}N\left(\mathrm{H}_{2}\right)_{\mathrm{ens}} \\
\mathrm{cm}^{-2}\end{array}$ & $\begin{array}{l}M_{\text {ens }} \\
M_{\odot}\end{array}$ & $\begin{array}{l}M_{\mathrm{cl}} \\
M_{\odot}\end{array}$ & $N_{\text {ens }}$ & ${\mathrm{C} / \mathrm{CO}_{\text {ens }}}$ & $\Phi_{\mathrm{A}}$ \\
& $4.0 \times 10^{21}$ & 2.9 & 0.001 & 2859 & 0.43 & 3.3 \\
$\left(10^{5}, 10^{2}\right)$ & $7.0 \times 0^{21}$ & 5.0 & 0.01 & 500 & 0.22 & 2.7 \\
& $1.2 \times 10^{22}$ & 8.6 & 0.1 & 86 & 0.11 & 2.2 \\
& $2.3 \times 10^{22}$ & 16.4 & 1.0 & 16 & 0.06 & 1.9 \\
& $4.0 \times 10^{22}$ & 28.6 & 10 & 3 & 0.03 & 1.6 \\
\hline & $2.0 \times 10^{21}$ & 1.4 & 0.001 & 1429 & 1.14 & 1.7 \\
$\left(10^{5}, 10^{3}\right)$ & $6.0 \times 10^{21}$ & 4.3 & 0.01 & 429 & 0.22 & 2.3 \\
& $1.1 \times 10^{22}$ & 7.9 & 0.1 & 79 & 0.13 & 2.0 \\
& $1.9 \times 10^{22}$ & 13.6 & 1.0 & 14 & 0.07 & 1.6 \\
& $3.7 \times 10^{22}$ & 26.4 & 10 & 3 & 0.04 & 1.6 \\
\hline & $3.0 \times 10^{21}$ & 2.1 & 0.1 & 21 & 0.9 & 2.4 \\
$\left(10^{4}, 10^{3}\right)$ & $6.0 \times 10^{21}$ & 4.3 & 1.0 & 4 & 0.4 & 2.2 \\
& $9.0 \times 10^{21}$ & 6.4 & 10 & 1 & 0.3 & 2.5 \\
\hline
\end{tabular}

* In Draine units.

$n=10^{5} \mathrm{~cm}^{-3}, \chi=10^{3}$ and $10^{2}$ and $n=10^{4} \mathrm{~cm}^{-3}$ and $\chi=10^{3}$. The drawn lines correspond to model calculations. We consider an ensemble of clumps with mass $M_{\mathrm{cl}}$ (also marked in Fig. 10) contributing to a total mass of $M_{\text {beam }}$ in a $60^{\prime \prime}$ beam. The total number of clumps in the beam is given by $N_{\mathrm{ens}}=M_{\text {beam }} / M_{\mathrm{cl}}$. Table 4 summarizes the defining parameters of the clump ensembles for which the $\mathrm{C} / \mathrm{CO}$ abundance ratios are shown in Fig. 10. We find that the observed $\mathrm{C} / \mathrm{CO}$ anti-correlation depends primarily on the characteristic mass of individual clump along a particular line of sight and does not have a strong dependence on the assumed FUV field. For clumps with densities of $10^{4} \mathrm{~cm}^{-3}$, masses below $0.1 M_{\odot}$ result in much higher values of $\mathrm{C} / \mathrm{CO}$ ratios than observed. The area filling factors $\left(\phi_{\mathrm{A}}\right)$ of the ensemble vary between 1.6 and 3.3 (Table 4), from the higher to the lower values of clump masses considered in our calculations. This indicates that the clumps fill the beam and in most cases partially overlap.

For the sake of completeness we also explored the second possible physical scenario, an inclined plane-parallel PDR, which might produce the observed uniform PDR emission apparently deep into the cloud and the observed $\mathrm{C} / \mathrm{CO}$ and $N\left(\mathrm{H}_{2}\right)$ anti-correlation. For this purpose we have used the plane-parallel PDR models illuminated from one side by Koester et al. (1994) in order to explicitly calculate the $\mathrm{C} / \mathrm{CO}$ ratios and $N\left(\mathrm{H}_{2}\right)$ for selected values of volume densities and UV-field. We have explored several values of $n$, slab-size $\left(N\left(\mathrm{H}_{2}\right)\right)$ and $\chi$ and have found consistently that for face-on PDRs the calculated $\mathrm{C} / \mathrm{CO}$ ratio drops much faster with $N\left(\mathrm{H}_{2}\right)$ as compared to the observations. Figure 10 shows the results of calculation for a planeparallel model with $n=2.5 \times 10^{4} \mathrm{~cm}^{-3}$ and $\chi=100$, for different slab-sizes or total $N\left(\mathrm{H}_{2}\right)$. Changing the inclination of the PDR has the same effect on both $N(\mathrm{C})$ and $N(\mathrm{CO})$, and thus the $\mathrm{C} / \mathrm{CO}$ ratio remains unaltered, though $N\left(\mathrm{H}_{2}\right)$ changes as the reciprocal of the sinus of the inclination angle. For this particular set of models we find that at $N\left(\mathrm{H}_{2}\right)=3 \times 10^{21} \mathrm{~cm}^{-2}$ the calculated $\mathrm{C} / \mathrm{CO}$ ratio is 0.08 . In contrast, observationally we find the $\mathrm{C} / \mathrm{CO}$ ratio to be 0.08 at $N\left(\mathrm{H}_{2}\right) \sim 3 \times 10^{22} \mathrm{~cm}^{-2}$. There is a discrepancy by a factor of 10 in $N\left(\mathrm{H}_{2}\right)$, which can be explained by an inclination angle of $6^{\circ}$. Similarly, different inclination angles are required to bring the different plane-parallel models to conform to the observed slope of the anti-correlation. Hence, though it might be possible to explain individual positions by making suitable choice of inclination angles, it is not possible to reproduce the observed anti-correlation at all positions in Cepheus B using a single inclination angle. Further, the anti-correlation as has been shown in Fig. 6 holds for a wide range of sources so it is rather unlikely that all of them would either have the same inclination angle or have different inclination angles and still conform to the same anti-correlation. The planeparallel models thus fail to reproduce the slope of the $\mathrm{C} / \mathrm{CO}$ vs. $N\left(\mathrm{H}_{2}\right)$ anti-correlation entirely which extends over two orders of magnitude in $N\left(\mathrm{H}_{2}\right)$. Intuitively, both uniformity of [C I] emission over the entire ridge of Cepheus $\mathrm{B}$ and the $\mathrm{C} / \mathrm{CO}$ vs. $N\left(\mathrm{H}_{2}\right)$ anti-correlation seen in most PDRs appear to be obvious outcomes of homogeneous plane-parallel models which are inclined. However, explicit calculations suggest that although the homogeneous plane parallel models predict almost constant $N(\mathrm{C})$ with changing $N\left(\mathrm{H}_{2}\right)$, they do not reproduce the observed global $\mathrm{C} / \mathrm{CO}$ vs. $N\left(\mathrm{H}_{2}\right)$ anti-correlation even when inclined.

We conclude that the spherical clumpy PDR model reproduces the frequently observed global $\mathrm{C} / \mathrm{CO}$ vs. $N\left(\mathrm{H}_{2}\right)$ anticorrelation over the entire range of observed $N\left(\mathrm{H}_{2}\right)$. These clumpy PDR models can thus be used to understand the typical density and size (hence mass) of the regions that dominate the observed emission. This does not imply the absence of clumps of sizes smaller or larger than those reproducing the observed $\mathrm{C} / \mathrm{CO}$ ratios. However the emission cannot be dominated by such clumps if the intensity and abundance ratios of these clumps differ significantly from the observed numbers. Hence the low observed relative abundance ratio $\mathrm{C} / \mathrm{CO}$ originate from positions of high column density where the emission is dominated by a few more massive clumps, while high ratios originate at positions covered by many small clumps, having a low line of sight column density.

The calculations presented here are meant to be of qualitative nature in order to derive insight into the clump parameters which determine the observed $\mathrm{C} / \mathrm{CO}$ ratio.

Figure 5 shows that the $\mathrm{C} / \mathrm{CO}$ ratio is higher towards the south-eastern part of the ridge further away from the sources of FUV radiation. This together with the results of clumpy PDR models which find that higher $\mathrm{C} / \mathrm{CO}$ ratios are associated with clumps of smaller mass present an interesting situation. In Cepheus B towards the north-western edge there are evidences of embedded sources while there are none to the south-east. Thus, using the clumpy PDR models we see that regions with embedded sources correspond to lines of sight with higher mass clumps.

\section{Summary}

We have presented the first largescale fully-sampled maps of [C I] (492 GHz) and ${ }^{12} \mathrm{CO} 4-3$, of the edge-on PDR, Cepheus B. The $[\mathrm{CI}$ ] emission does not delineate the PDR clearly, rather it traces the bulk emission of the more quiescent part of the molecular ridge extending to the east. Bulk of the PDR gas emits at velocities between -14 and $-11 \mathrm{~km} \mathrm{~s}^{-1}$. The ${ }^{12} \mathrm{CO} 4-3$ emission is strongly dominated by the hotspot present toward the northwestern tip of the molecular cloud. We observed ${ }^{12} \mathrm{CO} 4-3 / 1-0$ intensity ratios in excess of 1.5 towards the north-western edge of the cloud. Such ratios can only be explained in terms of temperature gradients along the line of sight. Excitation temperature, derived from the observed ${ }^{12} \mathrm{CO} 4-3 / 1-0$ ratio, varies between $20 \mathrm{~K}$ (in the quiescent parts of the cloud) to $50 \mathrm{~K}$ (close to the hotspot). We conclude that $[\mathrm{CI}]$ primarily traces the higher column density regions while ${ }^{12} \mathrm{CO} 4-3$ traces the higher temperature and density regions. 
In Cepheus B we find that $N(\mathrm{C})$ remains nearly constant although $N(\mathrm{CO})$ varies over one order of magnitude. The observed $\mathrm{C} / \mathrm{CO}$ ratio varies between 0.06 and 4.0 and shows a direct anticorrelation with the hydrogen column density $N\left(\mathrm{H}_{2}\right)$.

As estimated from the FIR continuum emission, the FUV radiation field in Cepheus B varies between 25 and 1500 in Draine units, with an average value of 100 over most of the [C I] emitting region. We have used the KOSMA- $\tau$ PDR model to explain the observed intensity ratios and the observed $\mathrm{C} / \mathrm{CO}$ vs. $N\left(\mathrm{H}_{2}\right)$ anti-correlation. These PDR model calculations show that for $\chi=100$, the observed $\mathrm{CI} /{ }^{13} \mathrm{CO} 2-1$ and $\mathrm{CI} /{ }^{12} \mathrm{CO} 4-3$ ratios indicate local hydrogen densities between $10^{4}-10^{5} \mathrm{~cm}^{-3}$ with even higher values at a few positions. The ${ }^{12} \mathrm{CO} 4-3 / 1-0$ ratio can be reproduced by the PDR models and the modelled clumps indeed exhibit outwardly increasing temperature gradients, as predicted from LTE analysis.

The observed global $\mathrm{C} / \mathrm{CO}$ vs. $N\left(\mathrm{H}_{2}\right)$ anti-correlation and its slope cannot be reproduced by single spherical PDR models or by plane-parallel homogeneous slabs with or without inclination. We were able to explain the observed anti-correlation between $\mathrm{C} / \mathrm{CO}$ and $N\left(\mathrm{H}_{2}\right)$ considering an ensemble of clumps of the same mass and density $\left(n=10^{5} \mathrm{~cm}^{-3}\right)$ for each observed position. We find that (i) the $\mathrm{C} / \mathrm{CO}$ ratio is characteristic of the dominant mass in the ensemble, clumps of smaller mass have higher $\mathrm{C} / \mathrm{CO}$ ratio and (ii) clumps of larger mass have to necessarily dominate emission along lines of sight with higher column density in order to reproduce the observed lower $\mathrm{C} / \mathrm{CO}$ ratio.

Acknowledgements. We thank Hans Ungerechts for allowing the use of the datasets from the Cepheus B on-the-fly project at the IRAM $30 \mathrm{~m}$ telescope. This material is based upon work supported by the Deutsche Forschungs Gemeinschaft (DFG) via grant SFB494 and the National Science Foundation under Grant No. AST-0228974. This research has made use of NASA's Astrophysics Data System.

\section{References}

Aumann, H. H., Fowler, J. W., \& Melnyk, M. 1990, AJ, 99, 1674 Bensch, F., Leuenhagen, U., Stutzki, J., \& Schieder, R. 2003, ApJ, 591, 1013 Beuther, H., Kramer, C., Deiss, B., \& Stutzki, J. 2000, A\&A, 362, 1109 Blaauw, A. 1964, ARA\&A, 2, 213

Boger, G. I., \& Sternberg, A. 2005, ApJ, 632, 302

Boreiko, R. T., Betz, A. L., \& Zmuidzinas, J. 1990, ApJ, 353, 181
Castets, A., Duvert, G., Dutrey, A., et al. 1990, A\&A, 234, 469

Cernicharo, J. 1985, ATM: A program to compute theoretical atmospheric opacity for frequencies $<1000 \mathrm{GHz}$, Tech. rep., IRAM

Draine, B. T. 1978, ApJS, 36, 595

Draine, B. T., \& Bertoldi, F. 1996, ApJ, 468, 269

Felli, M., Tofani, G., Harten, R. H., \& Panagia, N. 1978, A\&A, 69, 199

Gerin, M., Phillips, T. G., Keene, J., Betz, A. L., \& Boreiko, R. T. 1998, ApJ, 500,329

Graf, U. U., Heyminck, S, Michael, E. A., et al. 2002, in Millimeter and Submillimeter Detectors for Astronomy, ed. T. G. Phillips, \& J. Zmuidzinas, Proc. SPIE, Hawaii/USA, 4855, 322

Habing, H. J. 1968, Bull. Astr. Inst. Netherlands, 19, 421

Heyer, M. H., Brunt, C., Snell, R. L., et al. 1998, ApJS, 115, 241

Hiyama, S. 1998, Seitenbandkalibration radioastronomischer

Linienbeobachtungen, Diploma thesis, U. of Cologne

Hollenbach, D. J., \& Tielens, A. G. G. M. 1997, ARA\&A, 35, 179

Horn, J., Siebertz, O., Schmülling, F., et al.1999, Exp. Astron., 9, 17

Ikeda, M., Oka, T., Tatematsu, K., Sekimoto, Y., \& Yamamoto, S. 2002, ApJS, 139,467

Ingalls, J. G., Chamberlin, R. A., Bania, T. M., et al. 1997, ApJ, 479, 296

Kamegai, K., Ikeda, M., Malzaura, H., et al. 2003, ApJ, 589, 378

Kaufman, M. J., Wolfire, M. G., Hollenbach, D. J., \& Luhman, M. L. 1999, ApJ, 527,795

Kramer, C., \& Wild, W. 1994, IRAM Newsletter No. 18, 2

Kramer, C., Stutzki, J., \& Winnewisser, G. 1996, A\&A, 307, 915

Kramer, C., Degiacomi, C., Graf, U. U., et al. 1998, Proc. SPIE, 3357, 711

Kramer, C., Jakob, H., Mookerjea, B., et al. 2004, A\&A, 424, 887

Kramer, C., Mookerjea, B., Bayet, E., et al. 2005, A\&A, 441, 961

Koester, B., Stoerzer, H., Stutzki, J., \& Sternberg, A. 1994, A\&A, 284, 545

Malhotra, S., Kaufman, M. J., Hollenbach, O., et al. 2001, ApJ, 561, 766

Minchin, N. R., Ward-Thompson, D., \& White, G. J. 1992, A\&A, 265, 733

Nakagawa, T., Yui, Y. Y., Doi, Y., et al.. 1998, ApJS, 115, 259

Oka, T., Yamamoto, S., Iwata, M., et al. 2001, ApJ, 558, 176

Oka, T., Iwata, M., Maezawa, H., et al.. 2004, ApJ, 602, 80

Panagia, N., \& Thum, C. 1981, A\&A, 98, 295

Plume, R., Jaffe, D. T., Tatematsu, K., et al. 1999, ApJ, 512, 768

Röllig, M., Ossenkopf, V., Jeyakumar, S., \& Stutzki, J. 2006, A\&A, 451, 917

Sargent, A. I. 1977, ApJ, 218, 736

Sargent, A. I. 1979, ApJ, 233, 163

Schneider, N., Simon, R., Kramer, C., et al. 2003, A\&A, 406, 915

Sharpless, S. 1959, ApJS, 4, 257

Sternberg, A., \& Dalgarno, A. 1995, ApJS, 99, 565

Störzer, H., Stutzki, J., \& Sternberg, A. 1996, A\&A, 310, 592

Störzer, H., Zielinsky, M., Stutzki, J., \& Sternberg, A. 2000, A\&A, 358, 682

Testi, L., Olmi, L., Hunt, L., et al. 1995, A\&A, 303, 881

Ungerechts, H., et al. 2000, Imaging at Radio through Submillimeter Wavelengths, ASP Conf. Ser., 217, 190

Wilson, T. L., \& Rood, R. 1994, ARA\&A, 32, 191

Winnewisser, G., Bester, M., Ewald, R., Hilberath, W., \& Jacobs, K. 1986, A\&A, 167,207 